\title{
The cerebellum in cognitive processes: Supporting studies in children
}

\author{
MAJA STEINLIN \\ Neuropaediatrics, University Children's Hospital, Inselspital, Bern Switzerland
}

\begin{abstract}
Over the last decade, increasing evidence of cognitive functions of the cerebellum during development and learning processes could be ascertained. Posterior fossa malformations such as cerebellar hypoplasia or Joubert syndrome are known to be related to developmental problems in a marked to moderate extent. More detailed analyses reveal special deficits in attention, processing speed, visuospatial functions and language. A study about Dandy Walker syndrome states a relationship of abnormalities in vermis lobulation with developmental problems. Further lobulation or volume abnormalities of the cerebellum and/or vermis can be detected in disorders as fragile X syndrome, Downs's syndrome or William's syndrome. Neuropsychological studies reveal a relation of dyslexia and attention deficit disorder with cerebellar functions. These functional studies are supported by structural abnormalities in neuroimaging in these disorders. Acquired cerebellar or vermis atrophy was found in groups of children with developmental problems such as prenatal alcohol exposure or extreme prematurity. Also focal lesions during childhood or adolescence such as cerebellar tumour or stroke are related with neuropsychological abnormalities, which are most pronounced in visuo-spatial, language and memory functions. In addition, cerebellar atrophy was shown to be a bad prognostic factor considering cognitive outcome in children after brain trauma and leukaemia. In ataxia teleangiectasia, a neurodegenerative disorder affecting primarily the cerebellar cortex, a reduced verbal IQ and problems of judgment of duration are a hint of the importance of the cerebellum in cognition. In conclusion, the cerebellum seems to play an important role in many higher cognitive functions especially in learning. There is a suggestion that the earlier the incorrect influence the more pronounced the problems.
\end{abstract}

Key words: Cerebellum, cognition, neuropsychology, development, children

\section{Introduction}

Over the last three decades a lot of effort has been put into the detection of the non-motor cognitive functions of the cerebellum. Schmahmann and Sherman defined the cerebellar affective cognitive syndrome (1), a dysfunction that was shown in many other studies with patients suffering from different cerebellar abnormalities and lesions. There is growing evidence that the cerebellum is important for cognitive functions especially for executive, visuospatial and verbal functions (2). As parts of the motor functions of the cerebellum are within the area of learning or rather getting habituated for certain motor tasks, a role of the cerebellum for learning capacities also in cognitive functions was suggested. In a study of high functioning chess players and novices playing chess, a marked increase of cerebellar activity during a chess figure position task was shown in functional MR imaging, when novices were compared to high functioning chess players (3). This reflects the importance of the cerebellum in achieving some new, previously not exercised functions. As any child during development has a high need to achieve and improve new functions, there emerges the thought that the cerebellum might be extremely important during childhood, especially for the development of cognitive functions.

This review will concentrate on studies which support the importance of the cerebellum for the development of cognitive functions.

\section{Posterior fossa malformations}

Congenital malformations of the cerebellum are known to be related to developmental problems. In a series of 11 children with cerebellar hypoplasia Shevell et al. (4) reported their common initial presentation with developmental delay of mild to moderate degree. In our own series of 33 patients with congenital non-progressive ataxia, 12 had cerebellar hypoplasia (5). All our patients had delayed motor and speech development and cognitive developmental problems were obvious in 22 out of 34 patients. In 11 of these patients more extended neuropsychological examinations revealed IQ values ranging from 30 to 92 . In patients with better 
cognitive functions (IQ above 60) marked attention deficits, reduced processing speed and visuospatial problems were present. There was an asymmetrical profile with verbal functions being more superior than nonverbal functions. The neuropsychological profile suggests that possible longstanding deficits in visuospatial inputs during learning and the influence of impaired procedural learning might be important factors for cognitive problems of these children.

A further group of children with posterior malformation problems (vermis aplasia) and significant developmental problems are children with foubert syndrome. In our own study of long term follow-up, the children's cognitive developmental problems were obvious, with IQs ranging from $<30-85$ (6). More specific neuropsychological testing was not possible due to the marked motor and visual problems of these children. Since our publication in 1997, several groups have confirmed our initial data on intellectual deficits in Joubert syndrome $(7,8)$.

There are many other syndromes with moderate to marked cognitive developmental problems, where one of the features is a cerebellar abnormality. These data support the assumption that the cerebellum plays an important role in the development of children, not only for motor aspects but even more pronounced for cognitive abilities. There seems to be one exception: Children with Dandy Walker malformations are reported to have a normal cognitive development in about $50 \%$ of cases $(9,10)$. Boddaert et al revealed in their study $(9)$, that normal cognitive development in children with Dandy Walker malformation was related to normal vermian lobulation.

\section{Praenatal aetiology for cerebellar volume abnormalities}

Data from children with cerebellar hypoplasia, Joubert syndrome and also Dandy Walker syndrome point to the great importance of the vermis for the early development of cognitive functions. Structural imaging studies and especially volumetric studies support this assumption in several disorders, such as fragile $\mathrm{X}$ syndrome, dyslexia and ADHD.

In fragile $\mathrm{X}$ syndrome, there is a decrease of the posterior vermis, in female and male premutation carriers these vermal abnormalities are related with their cognitive involvement $(11,12)$.

Pinter et al. (13) showed that children with Down's syndrome have smaller cerebral volumes, and even smaller cerebellums. Chang et al. (14) suggested that preferential involvement of frontal lobe, cerebellum and mesial temporal lobe might correspond to the selective language and speech deficits in children with Down's syndrome.

In Williams's syndrome an enlarged cerebellum can be detected (15). Rae et al. (16) showed abnormalities in phosphor and proton MR spectroscopy in the cerebellum of children with Williams's syndrome and found a significant correlation between cerebellar ratios Cho/NA and Crea/NA and the ability of the subjects at various neuropsychological tests, including verbal and performance IQ as well as inspection time.

Interestingly, recent studies discuss that also in dyslexia cerebellar dysfunction seems to be present. The hypothesis of 'cerebellar dyslexia of Nicolson' suggests that cerebellar deficits at birth lead to phonological, speed and literacy problems through problems of articulation and working memory (17). This hypothesis is supported by recent findings of Stoodley (18) which show dyslexic adults to have an implicit learning problem, a function that is primarily processed by the cerebellum. In addition, the cerebellum is said to be one of the most consistent locations for structural differences between dyslexic and control participants in imaging studies (19). Moreover, Moretti et al. (20) published results of 10 patients with acquired vermian or paravermian lesions, all of which showed increased numbers of reading mistakes. In addition to cerebellar dysfunction, oculomotor abnormalities or disturbed cerebellar encephalic projections must also be considered to be related to these findings.

Attention deficit disorders (ADD) are another behavioural developmental problem which might have a relation to the cerebellum. Berquin et al. (21) analysed vermal volume and found it to be smaller in boys with ADHD. Abnormalities were mainly seen in the posterior lobe (VI-VIII). He suggests that a cerebellar-thalamo-prefrontal circuit dysfunction may subserve the motor control, inhibition and executive function deficits encountered in ADD. Similar data were found in many other studies. Functional MR studies give more evidence that ADD is a problem within the frontal lobe, however cerebellar-frontal connections are also prominent and therefore, exclusion of accompanying cerebellar problems is not possible. A functional MR study looking at baseline activity, showed bilateral cerebellar and vermis alteration in addition to frontal and parietal changes (22).

\section{Pre- and perinatally acquired cerebellar abnormalities}

Sowell et al. (23) were the first to discover that prenatal alcohol exposure leads to a decrease of anterior vermis volume, findings that have been confirmed by others $(24,25)$. O'Hare (26) showed that alcohol-exposed children had statistically significant reductions of the anterior vermis and posterior-inferior vermis and significant displacement of the anterior and posterior-inferior vermal regions. Anterior vermal dysmorphology was negatively correlated with verbal learning and 
memory performance within the alcohol-exposed group.

During the last few years more and more attention has been paid to the relationship of cerebellar lesions in preterm children and their cognitive outcome. Krägeloh Mann et al. reported preterm babies with marked cerebellar atrophy in 1999 and related this finding to severe developmental outcome (27). In 2002 , Johnsen et al. $(28,29)$ considered the possibility that extremely premature children might have injuries to the cerebellum, especially of inferior hemispheres and/or vermis. Accompanying lesions of the cerebrum were of less severe nature. The clinical features included motor impairment with variable degrees of ataxia, athethosis or dystonia and significant developmental problems for cognitive functions and language. Limperopoulos et al. (30) analysed the interaction between cerebrum and cerebellum in premature children suggesting that reduction of cerebellar volume and cerebral lesions are related to trophic transsynaptic effects. They state, that early-life cerebellar injury may contribute considerably to the high rates of cognitive, behavioural, and motor deficits reported for premature infants. Not only cerebellar atrophy is a signpost for possible cognitive problems in preterm children, but also cerebellar haemorrhage seems to have a similar effect in this group. In a recent study Limperopoulos (31) showed that neonatal mortality and morbidity in preterm children with cerebellar haemorrhage was significantly higher compared to preterm controls. Whether cerebellar haemorrhage points to a higher degree of perinatal damage or whether cerebellar haemorrhage alone worsens the outcome of these children remains to be answered in the future.

\section{Acquired cerebellar lesions during childhood}

All the above studies point to the importance of the cerebellum in the early cognitive development of children, but more detailed statements on exact dysfunctions are difficult to make in disorders leading to more generalized developmental problems in the majority of cases. Better models for these analyses are acquired focal cerebellar lesions. Cerebellar tumours, especially cerebellar astrocytomas, are suitable to study focal cerebellar lesions acquired during childhood. Levisohn (32) reported a group of 19 children with infratentorial tumours. They showed a specific pattern of neuropsychological problems with visuo-spatial, language sequencing and memory problems. These results could in large be confirmed by a study of Riva et al. (33) and our own study (34). Over the last few years, there have been published several reports of children after infratentorial tumour resection and their neuropsychological problems were described. The largest series were recently reported by Beebe et al. (35) who analysed neuropsychological problems in 103 children after tumour resection alone. They could not detect a specific profile of neuropsychological problems. Even in subgroups of location (right and left hemispheric tumours and vermis) no specific localisation related deficits were detected. However, as a group, these children showed widespread effects, most pronounced on performance IQ, spelling, adaptive behaviour and full scale IQ. Although a certain influence by motor difficulties could not be ruled out completely, motor problems could not explain all these abnormalities on its own. Concerning localization related data, controversial results were found by Scott et al. (36). In a small group of seven children greater damage to the right hemisphere led to a plateau in verbal and/or literacy skills and greater involvement of the left hemispheric cerebellum delayed or impaired non verbal/visuospatial skills. Another hint for the cognitive function of the cerebellum is the appearance of cerebellar mutism after infratentorial surgery (37), followed by dysarthria in the long term. Paquier et al. (37) suggested that the underlying pathophysiology for mutism is a disturbed mental initiation which precedes the programming of any intentional bucco-phonatory movements. In a case study of Kusano et al. (39) bilateral, but not unilateral, destruction of the dentate nuclei was followed by mutism. Papavasilou et al. (39) report on a case of mutism following diffuse cerebellitis. Focal lesions like stroke in the cerebellum are even better suited for characterizing specific cerebellar functions and dysfunctions respectively. In our own study of children after arterial ischaemic stroke we have followed so far only one child with pure cerebellar infarction. In a baseline neuropsychological assessment, this child has a neuropsychological profile like children with ADD, with an IQ value of 88 and significant problems in auditive short term memory and visuospatial functions (unpublished personal data). This supports not only the input of the cerebellum in cognitive functions, but also the possibility that the cerebellum plays an important role in problems of attention. There are several studies in adults confirming our observation. There is one report of a group of young patients with pure cerebellar infarcts, revealing also impairment in central aspects of attention and visuospatial skills (40). Due to their cognitive problems, two thirds of these patients could not return to work 12 months after the acute event.

Another model of cerebellar-acquired lesion is ataxia teleangiectasia, a heredodegenerative disease, which is known to affect primarily the cerebellar cortex. Mostofsky et al. (41) observed a significantly lower verbal IQ and, even corrected for their lower verbal IQ, a significant problem with judgement of duration in 17 patients with AT. These findings support the important role of the cerebellum in timing. 
In addition, there are several reports which show that neurocognitive problems after generalized brain events are related to cerebellar or vermis atrophy, as can be observed in children after head trauma (42) or in survivors of leukaemia (43).

\section{Conclusion}

The cerebellum seems to play an important role in many higher cognitive functions, while the earlier the incorrect influence occurs the more pronounced the problems will be. However, it is difficult to give more precise details on the most important cerebellar cognitive functions and how they are related to the functions of the cerebrum. Many of the abovesummarized problems are also related with cerebral structural or functional abnormalities or micro structural abnormalities of connections from cerebellum to pons or cerebrum. One also wonders, whether structural cerebellar abnormalities in a disorder like ADHD are primary problems or secondary atrophy due to functional problems of the cerebrum. In view of the wide variety of neuropsychological and speech problems related to the cerebellum (none of the functions unique to the cerebellum), the considerably more expressed developmental problems in children with primary cerebellar disorders compared to later acquired lesions, and also in view of the known cerebellar functions in learning, one might hypothesize that the most important or primary function of the cerebellum might be learning, either during development or later on during rehabilitation processes. Once the function of the cerebrum is well established, the importance of the cerebellum might decline. This hypothesis is also supported by the study of the chess players (3). The cerebrum might be like a glider, which needs the cerebellum as the motor aeroplane to bring it up in the air - once there, the glider is able to fly alone. But any troubles during this flight might bring it down again and the cerebellar motor aeroplane has to help once more to regain altitude!

\section{Acknowledgement}

I thank Sarah Walther for her help in preparing the manuscript.

\section{References}

1. Schmahmann JD, Sherman JC. The cerebellar cognitive affective syndrome. Brain. 1998;121:561-7.

2. Rapoport M, van Reekum R, Mayberg H. The role of the cerebellum in cognition and behaviour: a selective review. J Neuropsychiatry Clin Neurosci. 2000;12:193-8.

3. Campitelli G, Gobet F, Parker A. Structure and stimulus familiarity: a study of memory in chess-players with functional magnetic resonance imaging. Span J Psychol. 2005;8:238-45.

4. Shevell MI, Majnemer A. Clinical features of developmental disability associated with cerebellar hypoplasia. Pediatr Neurol. 1996;15:224-9.
5. Steinlin M, Styger M, Boltshauer E. Cognitive impairments in patients with congenital nonprogressive cerebellar ataxia. Neurology. 1999;53:966-73.

6. Steinlin M, Schmid M, Landau K, Boltshauser E. Follow-up in children with Joubert syndrome. Neuropediatrics. 1997;28:204-11.

7. Fenell EB, Gitten JC, Dede DE, Maria BL. Cognition, behavior, and development in Joubert syndrome. J Child Neurol. 1999;14:592-6.

8. Barreirinho MS, Teixeira J, Moreira NG, Bastos S, Goncalvez S, Barbot MC. Joubert's syndrome: report of 12 cases. Rev Neurol. 2001;32:812-7.

9. Boddaert N, Klein O, Ferguson N, Sonigo P, Parisot D, Hertz-Pannier L, et al. Intellectual prognosis of the DandyWalker malformation in children: the importance of vermian lobulation. Neuroradiology. 2003;45:320-4.

10. Gerszten PC, Albright AL. Relationship between cerebellar appearance and function in children with Dandy-Walker syndrome. Paediatr Neurosrurg. 1995;23:86-92.

11. Mostofksy SH, Mazzocco MM, Aakalu G, Warsofsky IS, Denckla MG, Reiss AL. Decreased cerebellar posterior vermis size in fragile $\mathrm{X}$ syndrome: correlation with neurocognitive performance. Neurology. 1998;50:121-30.

12. Cornish K, Kogan C, Turk J, Manly T, James N, Mills A, et al. The emerging fragile $\mathrm{X}$ premutation phenotype: evidence from the domain of social cognition. Brain Cogn. 2005;57:53-60.

13. Pinter JD, Eliez S, Schmitt JE, Capone CT, Reiss AL. Neuroanatomy of Down's syndrome: a high-resolution MRI study. Am J Psychiatry. 2001;158:1659-65.

14. Chang YC, Huanc CC, Huang SC. Volumetric neuroimaging in children with neurodevelopmental disorders - mapping the brain and behavior. Zhonghua Min Guo Xiao Er Ke Yi Xue Hui Za Zhi. 1998;39:285-92.

15. Jones W, Hesselink J, Courchesne E, Duncan T, Matsuda K, Bellugi U. Cerebellar abnormalities in infants and toddlers with Williams syndrome. Dev Med Child Neurol. 2002;44:688-94.

16. Rae C, Karmiloff-Smith A, Less MA, Dixon RM, Grant J, Blamire AM, et al. Brain biochemistry in Williams syndrome: evidence for a role of the cerebellum in cognition? Neurology. 1998;51:33-40.

17. Nicolson R, Fawcett AJ, Dean P. Dyslexia, development and the cerebellum. Trends Neurosci. 2001;24:515-6.

18. Stoodley CJ, Harrison EP, Stein JF. Implicit motor learning deficits in dyslexic adults. Neuropsychologia. 2006;44:795-8.

19. Eckert MA, Leonard CM, Richards RL, Aylward EH, Thomson J, Berninger VW. Anatomical correlates of dyslexia: frontal and cerebellar findings. Brain. 2003;126:482-94.

20. Moretti R, Bava A, Torre P, Antonello RM, Cazzato G. Reading errors in patients with cerebellar vermis lesions. J Neurol. 2002;249:461-8.

21. Berquin PC, Gliedd JN, Jacobsen LK, Hamburger SD, Krain AL, Rapoport JL, et al. Cerebellum in attention-deficit hyperactivity disorder: a morphometric MRI study. Neurology. 1998;50:1087-93.

22. Zang YF, Yong H, Chao-Zhe Z, Qing-Jiu C, Man-Qiu S, Meng $L$, et al. Altered baseline brain activity in children with ADHD revealed by resting-state functional MRI. Brain Dev. 2007;29:83-91.

23. Sowell ER, Jernigan TL, Mattson SN, Riley EP, Sobel DF, Jones KL. Abnormal development of the cerebellar vermis in children prenatally exposed to alcohol: size reduction in lobules I-V. Alcohol Clin Exp Res. 1996;20:31-4.

24. Autti-Ramo I, Autti T, Korkman M, Kettunen S, Salonen O, Valanne L. MRI findings in children with school problems who had been exposed prenatally to alcohol. Dev Med Child Neurol. 2002;44:98-106.

25. Roebuck TM, Mattson SN, Riley EP. A review of the neuroanatomical findings in children with fetal alcohol 
syndrome or prenatal exposure to alcohol. Alcohol Clin Exp Res. 1998;22:339-44.

26. O'Hare ED, Kan E, Yoshii J, Mattson SN, Riley EP, Thompson PM, et al. Mapping cerebellar vermal morphology and cognitive correlates in prenatal alcohol exposure. Neuroreport. 2005;16:1285-90.

27. Krägeloh-Mann I, Toft P, Lunding J, Andresen J, Pryds O, Lou HC. Brain lesions in preterms: origin, consequences and compensation. Acta Paediatr. 1999;88:897-908.

28. Johnsen SD, Tarby TJ, Lewis KS, Bird R, Prenger E. Cerebellar infarction: an unrecognized complication of very low birthweight. J Child Neurol. 2002;17:320-4.

29. Johnsen SD, Bodensteiner JB, Lotze TE. Frequency and nature of cerebellar injury in the extremely premature survivor with cerebral palsy. J Child Neurol. 2005;20:60-4.

30. Limperopoulos C, Soul JS, Haidar H, Huppi PS, Bassan H, Warfield SK, et al. Impaired trophic interactions between the cerebellum and the cerebrum among preterm infants. Pediatrics. 2005;116:844-50.

31. Limperopoulos C, Benson CB, Bassan H, Disalvo DN, Kinnamon DD, Moore M, et al. Cerebellar hemorrhage in the preterm infant: ultrasonographic findings and risk factors. Pediatrics. 2005;116:717-24.

32. Levisohn L, Cronin-Golomb A, Schmahmann JD. Neuropsychological consequences of cerebellar tumour resection in children: cerebellar cognitive affective syndrome in a paediatric population. Brain. 2000;123:1041-50.

33. Riva D, Giorgi C. The cerebellum contributes to higher functions during development: evidence from a series of children surgically treated for posterior fossa tumours. Brain. 2000;123:1051-61.

34. Steinlin M, Imfeld S, Zulauf P, Boltshauser E, Ridolfi-Lüthi A, Lövblad KO, Perrig W, Kaufmann F. Neuropsychological long term sequelae after posterior fossa tumor resection during childhood. Brain. 2003;126:1998-2008.
35. Beebe DW, Ris MD, Armstrong FD, Fontanesi J, Mulhern R, Holmes, et al. Cognitive and adaptive outcome in low-grade paediatric cerebellar astrocytomas: evidence of diminished cognitive and adaptive functioning in National Collaborative Research Studies (CCG 9891/POG 9130). J Clin Oncol. 2005;23:5198-204.

36. Scott RB, Stoodley CJ, Anslow P, Paul C, Stein JF, Sugden EM, et al. Lateralized cognitive deficits in children following cerebellar lesions. Dev Med Child Neurol. 2001;43:685-91.

37. Paquier $P$, van Mourik $M$, van Dongen $H$, CatsmanBerrevoets C, Brison A. Cerebellar mutism syndromes with subsequent dysarthria: a study of three children and a review of the literature. Rev Neurol. 2003;159:1017-27.

38. Kursano Y, Tanaka Y, Takasanua $\mathrm{H}$, Wada N, Tada $\mathrm{T}$, Kakizawa Y, Hongo $\mathrm{K}$. Transient cerebellar mutism caused by bilateral damage to the dentate nuclei after the second posterior fossa surgery. J Neurosurg. 2006;104:329-31.

39. Papavasilou AS, Kotsalis C, Trakadas S. Transient cerebellar mutism in the course of acute cerebellitis. Pediatr Neurol. 2004;30:71-4.

40. Malm J, Kristensen B, Karlsson T, Carlberg B, Fagerlund M, Olsson T. Cognitive impairment in young adults with infratentorial infarcts. Neurology. 1998;51:433-40.

41. Mostofsky SH, Kunze JC, Cutting LE, Lederman HM, Denckla MB. Judgment of duration in individuals with ataxiatelangiectasia. Dev Neuropsychol. 2000;17:63-74.

42. Soto Ares G, Vinchon M, Delmaire C, Abecidan E, Dheliemes P, Pruvo JP. Cerebellar atrophy after severe traumatic head injury in children. Childs Nerv Syst. 2001;17:263-9.

43. Ciesielski KT, Yanofsky R, Ludwig RN, Hill DE, Hart BL, Astur RS, et al. Hypoplasia of the cerebellar vermis and cognitive deficits in survivors of childhood leukemia. Arch Neurol. 1994;51:985-93. 